\title{
Detection of Colistin Resistance in Carbapenem Resistant Enterobacteriaceae by Reference Broth Microdilution and Comparative Evaluation of Three Other Methods
}

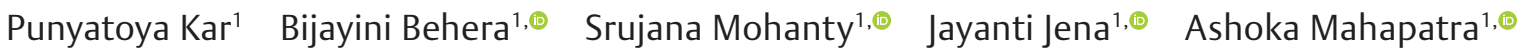

${ }^{1}$ Department of Microbiology, All India Institute of Medical Sciences, Bhubaneswar, Odisha, India

J Lab Physicians 2021;13:263-269.

\author{
Address for correspondence Ashoka Mahapatra, MD, Department \\ of Microbiology, All India Institute of Medical Sciences, \\ Bhubaneswar 751019, Odisha, India \\ (e-mail: micro_ashoka@aiimsbhubaneswar.edu.in, meetasoka@ \\ yahoo.co.in).
}

\begin{abstract}
Keywords

- agar dilution

- broth microdilution

- colistin

- colistin NP test

- E test

Objective Challenges in susceptibility testing of colistin along with increase in the prevalence of colistin-resistant carbapenemase-producing Enterobacteriaceae (CRE) pathogens needs addressal. Evaluation of user-friendly methods is necessary as an alternative to broth microdilution (BMD), the reference susceptibility testing method, for routine implementation in diagnostic clinical microbiology laboratories. Genotypic detection of the plasmid-mediated colistin resistance is also needed for infection control purposes.

Materials and Methods Colistin susceptibility of 200 nonduplicate clinical CRE isolates from December 2017 to June 2019 was determined by BMD, agar dilution (AD), E test, and rapid polymyxin NP test and interpreted as per the European Committee on Antimicrobial Susceptibility Testing. The results of AD, E test, and NP test were compared with that of BMD, considering minimal inhibitory concentration (MIC) $\leq 2 \mu \mathrm{g} / \mathrm{mL}$ as susceptible and $>2 \mu \mathrm{g} / \mathrm{mL}$ as resistant. Presence of any plasmid-mediated colistin resistance (mcr-1 and 2 ) was evaluated in 27 colistin-resistant CRE isolates by polymerase chain reaction.

Statistical Analysis Performance of different phenotypic methods was analyzed by comparing MIC results of $A D$ and $E$ test with that of reference BMD method. Agreement between BMD and the other two methods was expressed in terms of categorical agreement and essential agreement. Errors were expressed as very major error (VME: false-susceptible) and major error (ME: false-resistance) by $A D / E$ test. VME and ME of 3\% disagreement were considered unacceptable.

Results Colistin resistance was found in 27 (13.5\%) isolates by BMD method. The VME rates of both $A D(11 \%)$ and $E$ test $(37 \%)$ could not meet the Clinical and Laboratory Standards Institute recommendation ( $<3 \%$ VME rate is acceptable) as alternative tests to the reference BMD. Colistin NP test showed sensitivity and specificity of $85 \%$ and $98 \%$, respectively. The percentage discordant result in NP test was highest in Enterobacter spp. (17\%). None of the 27 colistin resistant isolates showed presence of mcr-1 and mcr-2 genes.
\end{abstract}

published online

July 6, 2021
DOI https://doi.org/ $10.1055 / \mathrm{s}-0041-1731137$ ISSN 0974-2727
(C) 2021. The Indian Association of Laboratory Physicians.

This is an open access article published by Thieme under the terms of the Creative Commons Attribution-NonDerivative-NonCommercial-License, permitting copying and reproduction so long as the original work is given appropriate credit. Contents may not be used for commercial purposes, or adapted, remixed, transformed or built upon. (https://creativecommons.org/licenses/by-nc-nd/4.0/).

Thieme Medical and Scientific Publishers Pvt. Ltd. A-12, 2nd Floor, Sector 2, Noida-201301 UP, India 
Conclusion High VME rate in $\mathrm{AD}$ and $\mathrm{E}$ tests precludes their use as alternatives to BMD for colistin susceptibility testing. NP test with moderate sensitivity but excellent specificity can be a good alternative for testing colistin susceptibility in CRE isolates, except in Enterobacter spp. Absence of mcr-1 and mcr-2 gene necessitates the exploration of other mechanisms of colistin resistance.

\section{Introduction}

Rapidly growing antimicrobial resistance (AMR) worldwide is compared with a potential tsunami aiming to crumble down the pillars of modern medicine. Around 700,000 annual deaths worldwide are attributed to AMR currently. This figure is predicted to rise to 10 million by 2050, with mortality attributed to AMR projected to outstrip all global major causes of death by $2050 .{ }^{1}$ Effective antimicrobial armamentarium has shrunken to combat the growing threat of carbapenemase-producing Enterobacteriaceae (CRE) in hospital settings. The revival of previously used antibiotics is regarded as one major strategy to combat AMR. In this context, old forgotten antimicrobial-like colistin has made an excellent comeback, with colistin enjoying a cult status, which was not the case in its first innings.

Colistin belongs to the cationic polypeptide antimicrobial, which act by binding with Lipid A of Gram-negative lipopolysaccharide resulting in loss of membrane integrity and cell death. Determination of accurate colistin susceptibility remains a challenge due to limiting factors like its multicomponent composition, poor diffusion to agar medium, synergistic effect with P-80, and adsorption to microtiter plates, which influence its performance in susceptibility testing. Though colistin has been used for such a long time in clinical fields, the optimal susceptibility testing method for colistin was undefined till recent years. In 2015, both the Clinical and Laboratory Standards Institute (CLSI) and the European Committee on Antimicrobial Susceptibility Testing (EUCAST) joint working group recommended the use of broth microdilution (BMD) as the reference method. ${ }^{2}$ However, in diagnostic clinical microbiology laboratories, the implementation of joint CLSI-EUCAST recommended BMD is difficult. Hence, the evaluation of other methods is of utmost necessity for therapeutic decision-making. Various other alternative methods that have been reported in literature include agar dilution (AD), E test, and colistin NP test.,

In the last decade, frequent usage of colistin against CRE had also led to the development of resistance against this drug. ${ }^{5}$ Colistin resistance was known to be caused by chromosomal mutation, but plasmid-mediated resistance (mediated by mor genes) has also been documented since $2015 .{ }^{6}$ Prevalence of colistin resistance varies according to geographical locations. In the SENTRY worldwide antimicrobial surveillance program conducted in 2009, percentage resistance to colistin in CRE isolates was around 0.67 to $1.6 \%$, with higher resistance rates noted in Enterobacter spp. (13.9-20.1\%), followed by Klebsiella pneumoniae (1.5-6.8\%) and Escherichia coli (0.2-0.6\%). ${ }^{7}$ The recent results of Antimicrobial Resistance Surveillance and Research Network of India (AMRSN) have reported colistin resistance rate in approximately $40 \%$ of carbapenem-resistant $K$. pneumoniae and in $10 \%$ of carbapenem resistant E. coli. ${ }^{8}$

Plasmid-mediated colistin resistance is a cause of concern as it can spread between bacteria of the same and different species. ${ }^{9}$ The location of $m c r-1$ gene on multidrug-resistance plasmids is alarming because the use of antimicrobials other than polymyxins can participate the co-selection of isolates carrying $m c r-1$ and in their spread. More worryingly, the plasmid-mediated mcr-1 gene has been identified in highly drug-resistant Enterobacteriaceae isolates harboring plasmids-encoding carbapenemase genes $\left(b l a_{\mathrm{KPC}-2}, b l a_{\mathrm{NDM}-1}\right.$, $b l a_{\mathrm{NDM}-5}, b l a_{\mathrm{NDM}-9}, b l a_{\mathrm{OXA}-48}$, and $\left.b l a_{\mathrm{VIM}-1}\right)$. Till date, several $m c r$ genes (mcr-1-mcr-8) have been identified from different places, but $m c r-1, m c r-1.2, m c r-2$, and $m c r-3$ are mostly studied in Enterobacteriaceae so far. ${ }^{10}$

In view of recent emergence of plasmid-mediated colistin resistance, the issues in colistin susceptibility testing and the need for an alternate more convenient testing method, we aimed to evaluate the performance of three phenotypic susceptibility methods (agar dilution, E test, and rapid colistin $\mathrm{NP}$ test) against the reference BMD and to detect the presence of plasmid-mediated colistin resistance ( $\mathrm{mcr}$ ) genes in CRE isolates at our hospital.

\section{Materials and Methods}

This prospective study was conducted on 200 clinically significant, consecutive, and nonduplicate CRE isolates recovered from patients receiving medical care at a tertiary care hospital of eastern India over a period of 2 years (January 2018-November 2019). Based on CLSI M100-S27, the isolates of Enterobacteriaceae demonstrating resistance to any one of the carbapenems, that is, ertapenem, imipenem, meropenem, and/or doripenem (10 $\mathrm{gg}$ ), were identified to be CRE. ${ }^{11}$ Susceptibility testing to colistin in these CRE isolates was performed primarily by the reference BMD test along with three other phenotypic methods, that is, $A D$, E test, and colistin NP test. E. coli NCTC 13846 (MIC: $4-8 \mu \mathrm{g} / \mathrm{mL}$ ) and $E$. coli ATCC 25922 (MIC: $0.25-2 \mu \mathrm{g} / \mathrm{mL}$ ) were used as positive and negative controls, respectively.

For BMD, stock solutions $(1,000 \mu \mathrm{g} / \mathrm{mL})$ of colistin sulfate salt (C4461 $=100 \mathrm{mg}$, Sigma-Aldrich) were prepared in distilled water, and desired concentration of working solutions $(0.25-8 \mu \mathrm{g} / \mathrm{mL})$ was made by twofold serial dilutions. To achieve $100 \mu \mathrm{L}$ of volume in each well of the U bottom 96-well polystyrene plate (Tarson Micro Test Plate), $25 \mu \mathrm{L}$ of drug, $25 \mu \mathrm{L}$ inoculum of concentration $\left(5 \times 10^{5} \mathrm{CFU} / \mathrm{mL}\right)$, and $50 \mu \mathrm{L}$ 
of double strength cation-adjusted Mueller Hinton (CAMH) broth were added and incubated at $35 \pm 2^{\circ} \mathrm{C}$ for 16 to 20 hours. The minimal inhibitory concentration (MIC) was recorded as the lowest concentration of drug that completely inhibited visible growth. ${ }^{12}$ MIC of positive and negative controls were ascertained before determining the MIC of test isolates.

For AD test, CAMH agar plates were prepared adding appropriate concentrations of colistin sulfate $(0.25-8 \mu \mathrm{g} / \mathrm{mL})$. Drug-free plates were used as growth controls. Test inoculums $\left(10^{4} \mathrm{CFU} / \mathrm{mL}\right)$ were spot inoculated on agar plates having different concentrations of colistin sulfate and incubated at $35 \pm 2^{\circ} \mathrm{C}$ for 16 to 20 hours. MIC was determined as the lowest concentration of drug that completely inhibited visible growth, disregarding a single colony or a faint haze caused by the inoculum. ${ }^{12}$

E test was performed by using Colistin Ezy MIC-strips (Himedia Pvt Ltd, Mumbai, India) as per manufacturer's instructions. The results of BMD, $\mathrm{AD}$, and $\mathrm{E}$ tests were interpreted by EUCAST recommended colistin break points for Enterobacteriaceae (sensitive: $\leq 2 \mu \mathrm{g} / \mathrm{mL}$, resistant: $>2 \mu \mathrm{g} / \mathrm{mL}$ ). ${ }^{2}$

\section{Rapid Colistin NP test}

This is a modification of polymyxin B NP test by Nordmann and Poirel. Colistin stock solution having $0.2 \mathrm{mg} / \mathrm{mL}$ colistin sulfate and rapid colistin NP solution $(\mathrm{pH}=6.7)$ having desired concentration of CAMH broth, phenol red, distilled water, and D-glucose were prepared. Test was performed in 96-well U-bottom sterile polystyrene microtiter plates with lids as described by Nordmann and Poirel Colistin resistance was interpreted by change in color of NP solution (original orange to yellow) due to change in $\mathrm{pH}$ as a result of bacterial growth in well-containing colistin. ${ }^{13}$

\section{Detection of Plasmid-Mediated Colistin Resistance}

All the CRE isolates with colistin MIC $>2 \mu \mathrm{g} / \mathrm{mL}$ were subjected for DNA extraction by using QIAGEN kit according to manufacturer's instructions. Uniplex conventional polymerase chain reaction (PCR) was performed by targeting mcr-1 (CLR F5'-CGGTCAGTCCGTTTGTTC-3', CLR R5'-CTTGGTCGGTCTGTAGGG-3') and mcr-2 (MCR2 IF 5'- TGTTGCTTGTGCCGATTGGA-3', MCR2 IR 5'-AGATGGTATTGTTGGTTGCTG-3') genes according to published methods. ${ }^{6,14}$ E. coli NCTC 13846 harboring mcr-1 gene was used as the positive control for $m c r-1$.

\section{Statistical Analysis}

For comparison of performance of different phenotypic methods, the MIC results of $\mathrm{AD}$ and $\mathrm{E}$ test were compared with that of reference BMD method. For ease of analysis, the MIC values between twofold dilutions in the E test were rounded off to the next twofold dilution (e.g., MIC $1.5 \mu \mathrm{g} / \mathrm{mL}$ was taken as $2 \mu \mathrm{g} / \mathrm{mL}$ ). Agreement between the reference test (BMD) and the other two methods were expressed in terms of categorical agreement (CA) and essential agreement (EA). CA was calculated as the percentage of isolates with result in the same category as the reference method, taking all isolates tested as denominator $(n=200)$. EA was calculated as the percentage of isolates that had MIC values within $\pm 1 \log _{2}$ dilution or \pm 1 twofold dilution of the reference standard. As per CLSI, any test having both CA and EA greater than $90 \%$ could be a reliable alternative to the reference test. Errors were ranked as very major error (VME: false-susceptible) and major error (ME: false-resistance) by $\mathrm{AD} / \mathrm{E}$-test. $\mathrm{VME}$ and $\mathrm{ME}$ of $\geq 3$ were considered unacceptable. ${ }^{15}$

\section{Results}

Among the 200 CRE isolates tested, E. coli was most common (113/200, 56.5\%), followed by $(79 / 200,39.5 \%)$ K. pneumoniae. Majority $(109,54.5 \%)$ were isolated from urine, followed by respiratory samples $(42,21 \%)$ and pus $(24,12 \%)$ (-Table 1). Susceptibility of the CRE isolates to various antimicrobials was as follows: amikacin (42,21\%), gentamicin (41, 20.5\%), netilmicin $(46,23 \%)$, ciprofloxacin (0\%), levofloxacin (4, 2\%), trimethoprim-sulfamethoxazole (31, 15.5\%), piperacillin-tazobactam (17, 8.5\%), cefoperazone-sulbactam (16, 8.0\%), and fosfomycin (11, 5.5\%). - Table 2 shows the MIC distribution of the CRE isolates to colistin by the various phenotypic methods of $\mathrm{BMD}, \mathrm{AD}$, and $\mathrm{E}$ test. In the BMD test, maximum $(130,65.5 \%)$ isolates demonstrated MIC of $\leq 0.25 \mu \mathrm{g} / \mathrm{mL}$, followed by $0.5 \mu \mathrm{g} / \mathrm{mL}(23,11.5 \%)$ and $1 \mu \mathrm{g} / \mathrm{mL}$ (17, 8\%), respectively. The $\mathrm{MIC}_{50}$ and $\mathrm{MIC}_{90}$ of the isolates were 0.25 and $8 \mu \mathrm{g} / \mathrm{mL}$, respectively ( - Table 2 ). In case of $\mathrm{AD}$, maximum isolates demonstrated MIC of $2 \mu \mathrm{g} / \mathrm{mL}$ (137, $68.5 \%$ ), followed by 1 and $>8 \mu \mathrm{g} / \mathrm{mL}$ (22 each, 11\%). Similarly, in E test, majority of isolates showed MIC of $1 \mu \mathrm{g} / \mathrm{mL}$ (126, 63\%), followed by $0.5 \mu \mathrm{g} / \mathrm{mL}(38,19 \%)$. The $\mathrm{MIC}_{50}$ and $\mathrm{MIC}_{90}$ in $\mathrm{AD}$ were 2 and $>8 \mu \mathrm{g} / \mathrm{mL}$, and in $\mathrm{E}$ test were 0.75 and $0.5 \mu \mathrm{g} / \mathrm{mL}$, respectively.

Table 1 Sources of carbapenemase-producing Enterobacteriaceae

\begin{tabular}{|l|l|l|l|l|l|l|}
\hline \multirow{2}{*}{ Organism } & \multicolumn{5}{|c|}{ Source } & Total \\
\cline { 2 - 8 } & $\begin{array}{l}\text { Urine } \\
(\boldsymbol{n}=\mathbf{1 0 9})\end{array}$ & $\begin{array}{l}\text { Pus } \\
(\boldsymbol{n}=\mathbf{2 4 )}\end{array}$ & $\begin{array}{l}\text { Respiratory } \\
(\boldsymbol{n}=\mathbf{4 2})\end{array}$ & $\begin{array}{l}\text { Blood } \\
(\boldsymbol{n}=\mathbf{0 5})\end{array}$ & $\begin{array}{l}\text { Miscellaneous } \\
(\boldsymbol{n}=\mathbf{2 0})\end{array}$ & $\boldsymbol{n}=\mathbf{2 0 0}$ \\
\hline Escherichia coli & 74 & 14 & 12 & 01 & 12 & 113 \\
\hline Klebsiella pneumoniae & 31 & 09 & 25 & 04 & 08 & 79 \\
\hline Enterobacter spp. & 04 & 01 & 03 & & & 08 \\
\hline
\end{tabular}

ancludes isolates from body fluids, tissue and catheter tips. 
Table 2 Distribution of colistin minimal inhibitory concentration in carbapenemase-producing Enterobacteriaceae isolates by different methods

\begin{tabular}{|c|c|c|c|c|c|c|c|c|c|c|}
\hline \multirow[t]{3}{*}{ Organisms } & \multirow{3}{*}{$\begin{array}{l}\text { No. of } \\
\text { isolates }\end{array}$} & \multicolumn{7}{|c|}{ Colistin MIC ( $\mu \mathrm{g} / \mathrm{mL})$} & \multirow{3}{*}{$\begin{array}{l}\mathrm{MIC}_{50} \\
\mu \mathrm{g} / \\
\mathrm{mL}\end{array}$} & \multirow{3}{*}{$\begin{array}{l}\mathrm{MIC}_{90} \\
\mu \mathrm{g} / \mathrm{mL}\end{array}$} \\
\hline & & $\leq 0.25$ & 0.5 & 1 & 2 & 4 & 8 & $>8$ & & \\
\hline & & \multicolumn{7}{|c|}{ Broth microdilution test } & & \\
\hline Escherichia coli & 113 & 93 & 07 & 09 & 01 & 02 & & 01 & $\leq 0.25$ & 1 \\
\hline $\begin{array}{l}\text { Klebsiella } \\
\text { pneumoniae }\end{array}$ & 79 & 33 & 15 & 08 & 01 & 04 & 06 & 12 & 0.5 & $>8$ \\
\hline Enterobacter spp. & 08 & 04 & 01 & & 01 & & & 02 & $\leq 0.25$ & $>8$ \\
\hline \multirow[t]{2}{*}{ Total } & 200 & $\begin{array}{l}130 \\
(65.5 \%)\end{array}$ & $\begin{array}{l}23 \\
(11.5 \%)\end{array}$ & $\begin{array}{l}17 \\
(8 \%)\end{array}$ & $\begin{array}{l}03 \\
(1.5 \%)\end{array}$ & $\begin{array}{l}06 \\
(3 \%)\end{array}$ & $\begin{array}{l}06 \\
(3 \%)\end{array}$ & $\begin{array}{c}15 \\
(7.5 \%)\end{array}$ & 0.25 & $>8$ \\
\hline & & \multicolumn{7}{|c|}{ Agar dilution test } & & \\
\hline Escherichia coli & 113 & 00 & 00 & 18 & 91 & 03 & 00 & 01 & 2 & 2 \\
\hline $\begin{array}{l}\text { Klebsiella } \\
\text { pneumoniae }\end{array}$ & 79 & 00 & 00 & 02 & 42 & 16 & 00 & 19 & 2 & $>8$ \\
\hline Enterobacter spp. & 08 & 00 & 00 & 02 & 04 & 00 & 00 & 02 & 2 & $>8$ \\
\hline \multirow[t]{2}{*}{ Total } & 200 & 00 & 00 & $\begin{array}{l}22 \\
(11 \%)\end{array}$ & $\begin{array}{l}137 \\
(68.5 \%)\end{array}$ & $\begin{array}{l}19 \\
(9.5 \%)\end{array}$ & 00 & $\begin{array}{c}22 \\
(11 \%)\end{array}$ & 2 & $>8$ \\
\hline & & \multicolumn{7}{|c|}{ E test } & & \\
\hline Escherichia coli & 113 & 00 & 25 & 78 & 09 & 00 & 00 & 01 & 0.75 & 1 \\
\hline $\begin{array}{l}\text { Klebsiella } \\
\text { pneumoniae }\end{array}$ & 79 & 01 & 12 & 43 & 08 & 05 & 02 & 08 & 1 & 6 \\
\hline Enterobacter spp. & 08 & 01 & 01 & 05 & 00 & 00 & 00 & 01 & 0.75 & 1 \\
\hline Total & 200 & $\begin{array}{l}02 \\
(1 \%)\end{array}$ & $\begin{array}{l}38 \\
(19 \%)\end{array}$ & $\begin{array}{l}126 \\
(63 \%)\end{array}$ & $\begin{array}{l}17 \\
(8.5 \%)\end{array}$ & $\begin{array}{l}05 \\
(2.5 \%)\end{array}$ & $\begin{array}{c}02 \\
(1 \%)\end{array}$ & $\begin{array}{l}10 \\
(5 \%)\end{array}$ & 0.75 & 256 \\
\hline
\end{tabular}

Abbreviation: MIC, minimal inhibitory concentration.

Table 3 Categorical agreement, essential agreement, very major error, and major error rates of agar dilution, E test in comparison to broth microdilution

\begin{tabular}{|l|l|l|l|l|l|l|l|l|}
\hline Methods & \multicolumn{2}{|l|}{$\begin{array}{l}\text { No. (\%) of isolates } \\
(n=200)\end{array}$} & \multicolumn{3}{|c|}{ No. (\%) of isolates with } & $\begin{array}{l}\text { CLSI } \\
\text { acceptable } \\
\text { limits of } \\
\text { VME and } \\
\text { ME }\end{array}$ & Remarks \\
& Susceptible & Resistant & EA & CA & ME & VME & & \\
\hline BMD & $173(86.5)$ & $27(13.5)$ & & & & & & Unacceptable \\
\hline AD & $176(88)$ & $24(12)$ & $44 / 200(22)$ & $181 / 200(90.5)$ & $\begin{array}{l}16 \\
(9.2)\end{array}$ & $3(11)$ & $<3 \%$ & Unacceptable \\
\hline E test & $183(91.5)$ & $17(8.5)$ & $85 / 200(42.5)$ & $195 / 200(95)$ & 0 & $\begin{array}{l}10 \\
(37)\end{array}$ & $<3 \%$ \\
\hline
\end{tabular}

Abbreviations: AD, agar dilution; BMD, broth microdilution; CA, categorical agreement; CLSI, Clinical and Laboratory Standards Institute; EA, essential agreement; MIC, minimal inhibitory concentration; ME, major error; VME, very major error.

Colistin resistance was detected in 27 (13\%, 27/200) CRE isolates as per the reference BMD method, and majority (22/27, 81\%) were K. pneumoniae, followed by E. coli $(3 / 27$, $11 \%)$. On comparison of MIC values of AD and $E$ test with that of BMD in these 27 colistin-resistant isolates, it was found that $3(3 / 27,11 \%)$ were sensitive to colistin ( $\mathrm{MIC}=2 \mu \mathrm{g} / \mathrm{mL}$ ) by the $\mathrm{AD}$ test, whereas $10(10 / 27,37 \%)$ were sensitive to colistin (MIC $\leq 2 \mu \mathrm{g} / \mathrm{mL}$ ) by E test ( - Table 3 ). On the other hand, out of the 173 colistin-susceptible isolates by BMD, 16 (16/173, 9.2\%) demonstrated resistance by $\mathrm{AD}$ while none demonstrated resistance by the E test. Thus, the false-susceptible or very major error rate in $\mathrm{AD}$ and $\mathrm{E}$ test was 11 and $37 \%$, while false-resistant or major error rate was 9.2 and $0 \%$, respectively (-Table 3). AD method showed a CA of $90.5 \%$ (181/200) and EA of 22\% (44/200) compared with BMD, while E test showed a CA of $95 \%$ (190/200) and EA of $42.5 \%$ (85/200), respectively (- Table 3). Further analysis showed that the colistin MIC values by the AD method were found to be exactly the same as BMD in 21 isolates ( - Fig. 1, - Table 4). Of the rest 179 isolates, 23 had MIC within $\pm 1 \log$, dilutions by AD compared with BMD, while 37 and 119 isolates exhibited two and three higher $\log _{2}$ dilution higher colistin MICs 


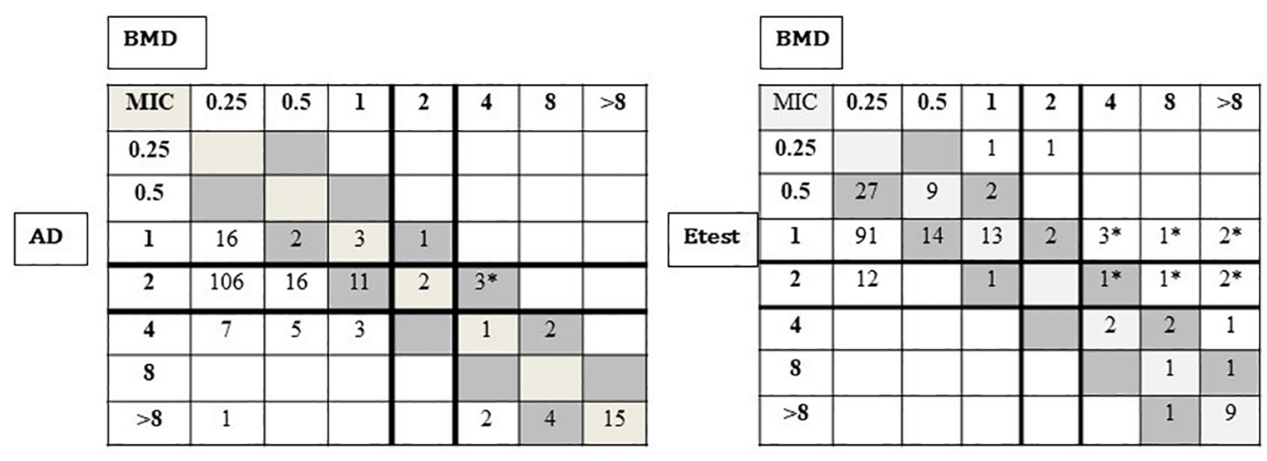

Fig. 1 Scattergrams showing numbers of isolates $(n=200)$ with colistin minimal inhibitory concentration determined by agar dilution, E test versus broth microdilution as a reference method. Solid lines represent the 2015 European Committee on Antimicrobial Susceptibility Testing breakpoint for susceptibility ( $\geq 2 \mu \mathrm{g} / \mathrm{mL}$ ). The diagonal boxes (light gray and dark gray) indicate categorical agreement and essential agreement. Very major errors are indicated by *.

Table 4 Differences in $\log _{2}$ dilutions of minimal inhibitory concentrations obtained by agar dilution, E test compared with broth microdilution

\begin{tabular}{|c|c|c|c|c|c|c|c|}
\hline \multirow[t]{2}{*}{ Method } & \multicolumn{7}{|c|}{ No. $(\%)$ of isolates showing MIC difference ( $\log _{2}$ dilution) of: } \\
\hline & -3 & -2 & -1 & 0 & +1 & +2 & $\geq+3$ \\
\hline$A D$ & 0 & 0 & 6 & 21 & 17 & 37 & 119 \\
\hline E test & 6 & 6 & 8 & 34 & 43 & 91 & 12 \\
\hline
\end{tabular}

Abbreviations: AD, agar dilution; MIC, minimal inhibitory concentration.

in AD compared with BMD (-Fig. 1, - Table 4). As regards E test, 34 isolates had the same MIC in E test as that of BMD, while 51 had MIC within $\pm 1 \log _{2}$ dilutions by both E test and $\mathrm{BMD}$, and 115 isolates exhibited both higher and lower colistin MICs by \pm 2 and $\pm 3 \log _{2}$ dilutions by E test compared with reference BMD ( - Fig. 1, - Table 4 ).

The rapid colistin NP test showed an overall concordance of $96 \%$ with the BMD test, as it was observed that $11.5 \%$ (23/200) and $84.5 \%$ (169/200) isolates exhibited similar resistance and susceptibility results to colistin by both rapid colistin NP and BMD tests. The sensitivity, specificity, positive predictive value, and negative predictive values of the rapid colistin NP test was $85.1,97.6,85$, and $97 \%$, respectively. The discordant results by rapid colistin NP test of the eight organisms were analyzed in different genera which showed $12.5 \%$ (1/8) in Enterobacter spp., 4.4\% (5/113) in E. coli, and 2.5\% (2/79) in K. pneumoniae isolates.

The ME and VME could not be derived in rapid colistin NP test as there is no absolute MIC value in this test. None of the 27 colistin-resistant CRE isolates were positive for $m c r-1$ and mcr-2 genes.

\section{Discussion}

Emergence of carbapenem and colistin resistant pathogens in clinical settings and challenges in susceptibility testing of colistin are leading to a catastrophic situation in treating serious infections. In diagnostic clinical microbiology laboratories, the implementation of joint CLSI-EUCAST recommended BMD is difficult and hence necessitates the evaluation of other methods for therapeutic decision-making. The determination of plasmid-mediated colistin resistance mechanism is also equally important for infection control purpose. In this context, this prospective study was undertaken to determine colistin susceptibility in 200 CRE isolates primarily by reference BMD method, and three other methods, namely AD, E test, and rapid colistin NP test, were compared with the BMD. Colistin-resistant isolates were further screened for the presence of $m c r-1$ and $m c r-2$ by PCR.

In this study, 54.5\% (109/200) CRE isolates were recovered from urine, followed by respiratory $(42 / 200,21 \%)$ and pus $(24 / 200,12 \%)$ samples, and the predominant CRE was E. coli (56.5\%), followed by K. pneumoniae (39.5\%) and Enterobacter species (4\%). Similar findings have been observed in Indian studies by Yadav et al, and Saeed et al as well as in a multicentric study from United States that have reported the predominance of $E$. coli among CRE in hospital based studies from Haryana and three Boston hospitals. ${ }^{16,17,18}$

However, 13.5\% (27/100) of the CRE isolates were resistant to colistin by reference BMD method. Colistin resistance is showing an increasing trend across the world, and the prevalence varies according to geographical locations. A very high rate (20-40\%) has been observed among carbapenem-resistant K. pneumonia (CRKP) in Italy and Greece. ${ }^{19}$ The recent results of AMRSN have reported colistin resistance rate in approximately $40 \%$ of CRKP and 10\% of carbapenem-resistant E. coli (CREC).$^{8}$ In our study, the frequency of colistin resistance in CRKP was 27.8\% (22/79) and CREC was 2.7\% (3/113), which is comparatively lower to that of AMRSN. But our frequency of CRKP was higher when compared with the study at CMC Vellore (15\% of CRKP). ${ }^{20}$ Presence of capsular polysaccharides (CPS) and increased expression of efflux pumps are the additional contributing factors for high frequency of colistin resistance in CRKP compared with CREC. ${ }^{21}$

In our study, $13.5 \%(27 / 200)$ isolates were resistant to colistin by reference BMD method (MIC $\leq 2 \mu \mathrm{g} / \mathrm{mL}$ ), with 
highest prevalence $(81 \%)$ noted in K. pneumoniae. The colistin $\mathrm{MIC}_{90}$ values in K. pneumoniae were higher $(>8,8$, and $6 \mu \mathrm{g} / \mathrm{mL})$ compared with that of $E$. coli $(1,2$, and $1 \mu \mathrm{g} / \mathrm{mL})$ in all the three methods (BMD, AD, and E test, respectively). This higher MIC range in K. pneumoniae could be due to the presence of CPS contributing to colistin resistance.$^{21}$

Regarding agreement of E test, although it had a CA of $95 \%$, the essential agreement was only $42.5 \%$. A twofold and threefold higher MIC were observed in $45.5 \%$ (91/200) and 6\% (12/200) of the CRE isolates respectively by the $\mathrm{E}$ test. This overestimation of colistin MIC by E test was previously supported by Bakthavatchalam et al, Singhal et al, and Chew et al. ${ }^{21,22,23}$ This poor correlation of $\mathrm{E}$ test with reference BMD could be attributed to the poor diffusion of colistin into the agar medium. The VME of colistin E test was $37 \%$ in our study, which correlates well to the reports of Bakthavatchalam et al and Chew et al where VME of E test was 42 and $12 \%$, respectively. The results of all these studies indicate that colistin $\mathrm{E}$ test VME $>3 \%$ and thus could not meet the CLSI recommendation as an alternative to the reference BMD test.

Regarding agreement of $\mathrm{AD}$ test, it showed a CA of $90.5 \%$ and EA of $22 \%$ as compared with the reference BMD. Furthermore, 59.5\% (119/200) of the CRE isolates showed a threefold and $18.5 \%$ (37/200) showed a twofold higher colistin MIC by AD. The overestimation of colistin MIC value by $\mathrm{AD}$ is previously described by Dafopoulou et al, where $29.5 \%$ isolates had twofold higher MIC by AD. ${ }^{24}$ The VME and ME of $\mathrm{AD}$ in our study was 11 and $9.2 \%$, respectively. This is in concordance with the observation by Ramanan et al, where the EA, VME, and ME of AD were $90,2.5$, and $2.9 \%$, respectively. ${ }^{25}$

The rapid colistin NP test had a specificity of $97.6 \%$ but the sensitivity was $85 \%$. This low sensitivity in our study was due to the observation of false negative results in Enterobacter isolates (12.5\%) by the colistin NP test. Simar et al had also reported a low sensitivity of approximately $25 \%$ in Enterobacter isolates due to false negative results by rapid colistin NP test and attributed to heteroresistance. ${ }^{26}$

The mcr-1 and mcr-2 genes were not detected in any of the 27 colistin-resistant isolates. This could be either due to the presence of other plasmid-mediated resistance mechanisms ( $m c r-3-m c r-8)$ or chromosomal-mediated resistance. Similar observation has been reported by Pragasam et al, where the whole genome sequence analysis on eight colistin-resistant isolates of $K$. pneumonia revealed the mutation of $m g r B$ gene with absence of $m c r-1$ and $m c r-2$ genes.$^{27}$ In India, till date, there is only one report of $m c r-1$ gene in a clinical isolate of $E$. coli in 2016. ${ }^{28}$ Another study from India reported the absence of plasmid mediated $m c r-1$ and $m c r-2$ genes, whereas presence of $m g r B$ gene (chromosomal mediated) was confirmed in 4 out of 17 colistin-resistant isolates of K. pneumoniae. ${ }^{29}$ Regarding the susceptibility profile of the CRE isolates to other antimicrobials in our study, most of them were MDR (nonsusceptibility to at least one agent in three or more antimicrobial categories). On the other hand, 100\% CRE isolates did not have activity against fluoroquinolones, 30 to $35 \%$ of carbapenem-resistant E. coli were susceptible to aminoglycosides, but other CRE were resistant.
Limitations in this study was molecular detection of colistin resistance only included plasmid-mediated resistance. Moreover, clinical details of patients prior colistin therapy, adequacy of dosing, and outcome of patients harboring colistin-resistant isolates could not be analyzed. This is a single-center study with limited number of CRE isolates for a period of 2 years. Hence, future large-scale studies over years are necessary to understand the epidemiology and evolution of colistin resistance.

\section{Conclusion}

Colistin resistance was detected in $13.5 \%$ of CRE in this by reference BMD method. K. pneumoniae was the major colistin-resistant isolate (81\%). The VME rates of AD (11\%) and $\mathrm{E}$ test $(37 \%)$ precludes the use of these tests as alternative to reference BMD for the determination of colistin susceptibility. Rapid NP test had excellent specificity (97/6\%), though the sensitivity was low (85\%). None of the 27 colistin-resistant CRE isolates were positive for plasmid-mediated $m c r-1$ and mcr-2 genes.

\section{Funding}

None.

\section{Conflict of Interest}

None declared.

\section{Acknowledgments}

The authors acknowledge the Tata Medical Centre-Cancer Hospital and Research Center, Kolkata, for timely help in providing us the reference strain Escherichia coli (mcr-1) NCTC 13846, needed in our study.

\section{References}

1 The Review on Antimicrobial Resistance, chaired by Jim O'Neill. Antimicrobial resistance: tackling a crisis for the health and wealth of nations. Available at: http://www. jpiamr.eu/wp-content/uploads/2014/12/AMR-Review-PaperTackling-a-crisis-for-the-health-and-wealth-of-nations_1-2. pdf. Accessed December 2014

2 Recommendations for MIC determination of colistin (polymyxin E) as recommended by the joint CLSI-EUCAST Polymyxin breakpoints Working Group. Available at: http:// www.bioconnections.co.uk/files/merlin/Recommendations_ for_MIC_determination_of_colistin_March_2016.pdf. Accessed 2016

3 Manohar P, Shanthini T, Ayyanar R, et al. The distribution of carbapenem- and colistin-resistance in Gram-negative bacteria from the Tamil Nadu region in India. J Med Microbiol 2017;66(7):874-883

4 Arjun R, Gopalakrishnan R, Nambi PS, Kumar DS, Madhumitha R, Ramasubramanian V. A study of 24 patients with colistin-resistant gram-negative isolates in a tertiary care hospital in South India. Indian J Crit Care Med 2017;21(5):317-321

5 Berglund B. Acquired resistance to colistin via chromosomal and plasmid-mediated mechanisms in Klebsiella pneumoniae. Infect Microbes Dis. 2019;1:10

6 Liu Y-Y, Wang Y,Walsh TR, et al.Emergence of plasmid-mediated colistin resistance mechanism MCR-1 in animals and human 
beings in China: a microbiological and molecular biological study. Lancet Infect Dis 2016;16(2):161-168

7 Gales AC, Jones RN, Sader HS. Contemporary activity of colistin and polymyxin B against a worldwide collection of Gram-negative pathogens: results from the SENTRY Antimicrobial Surveillance Program (2006-09) J Antimicrob Chemother 2011;66(9):2070-2074

8 Walia K, Madhumathi J, Veeraraghavan B, et al. Establishing antimicrobial resistance surveillance \& research network in India: journey so far. Indian J Med Res 2019;149(2):164-179

9. Büchler AC, Gehringer C, Widmer AF, et al. Risk factors for colistin-resistant Enterobacteriaceae in a low-endemicity setting for carbapenem resistance: a matched case-control study. Euro Surveill 2018;23(30):1700777.

10. Bakthavatchalam YD, Pragasam AK, Biswas I, Veeraraghavan B. Polymyxin susceptibility testing, interpretative breakpoints and resistance mechanisms: an update. J Glob Antimicrob Resist 2018;12:124-136

11 CLSI, Performance Standards for Antimicrobial Susceptibility Testing; Twenty-Second Informational Supplement. CLSI document M100-S27. Wayne, PA: Clinical and Laboratory Standards Institute; 2017

12 CLSI, Methods for Dilution Antimicrobial Susceptibility Tests for Bacteria That Grow Aerobically; Approved Standard. 10th edition. CLSI document M07-A10. Wayne, PA: Clinical and Laboratory Standards Institute; 2015

13 Nordmann P, Jayol A, Poirel L. Rapid Detection of Polymyxin Resistance in Enterobacteriaceae. Emerg Infect Dis 2016;22(6):1038-1043

14 Xavier BB, Lammens C, Ruhal $\mathrm{R}$, et al. Identification of a novel plasmid-mediated colistin-resistance gene, mcr-2, in Escherichia coli, Belgium, June 2016. Euro Surveill 2016;21(27):30280

15 Humphries RM, Ambler J, Mitchell SL, et al . CLSI methods development and standardization working group best practices for evaluation of antimicrobial susceptibility tests J Clin Microbiol2018;DOI:10.1128/JCM.01934-17

16 Yadav S. Prevalence of carbapenem resistant Enterobacteriaceae at a tertiary care institute: Need for policy. Int J Curr Res 2018;DOI:10.7860/JCDR/2021/47332.14627

17 Saeed NK, Alkhawaja S, Azam NFAEM, Alaradi K, Al-Biltagi M. Epidemiology of carbapenem-resistant Enterobacteriaceae in a tertiary care center in the Kingdom of Bahrain. J Lab Physicians 2019;11(2):111-117

18 Cerqueira GC, Earl AM, Ernst CM, et al. Multi-institute analysis of carbapenem resistance reveals remarkable diversity, unexplained mechanisms, and limited clonal outbreaks. Proc Natl Acad Sci U S A 2017;114(5):1135-1140

19 Amladi AU, Abirami B, Devi SM, et al. Susceptibility profile, resistance mechanisms \& efficacy ratios of fosfomycin, nitrofurantoin \& colistin for carbapenem-resistant Enterobacteriaceae causing urinary tract infections. Indian J Med Res 2019;149(2):185-191

20 Srinivasan VB, Rajamohan G. KpnEF, a new member of the Klebsiella pneumoniae cell envelope stress response regulon, is an SMR-type efflux pump involved in broad-spectrum antimicrobial resistance. Antimicrob Agents Chemother 2013;57(9):4449-4462

21 Bakthavatchalam YD, Shankar A, Thukaram B, Krishnan DN, Veeraraghavan B. Comparative evaluation of susceptibility testing methods for colistin and polymyxin B among clinical isolates of carbapenem- resistant Klebsiella pneumoniae and Acinetobacter baumannii. J Infect Dev Ctries 2018;12(6):504-507

22 Singhal L, Sharma M, Verma S, et al. Comparative evaluation of broth microdilution with polystyrene and glass-coated plates, agar dilution, E-test, Vitek, and disk diffusion for susceptibility testing of colistin and polymyxin b on carbapenem-resistant clinical isolates of acinetobacter baumannii. Microb Drug Resist 2018;24(8):1082-1088

23 Chew KL, La M-V, Lin RTP, Teo JWP, Colistin and polymyxin B susceptibility testing for carbapenem-resistant and mcr-positive enterobacteriaceae: comparison of Sensititre, MicroScan, Vitek 2 and E test with Broth Microdilution J Clin Microbiol2017;2609-1616

24 Dafopoulou K, Zarkotou O, Dimitroulia E, et al. Comparative evaluation of colistin susceptibility testing methods among carbapenem-nonsusceptible klebsiella pneumoniae and acinetobacter baumannii clinical isolates. Antimicrob Agents Chemother 2015;59(8):4625-4630

25 Ramanan P, Cole N, Kohner P, Uhl J, Patel R, Schuetz A. Colistin susceptibility testing of enterobacteriaceae by agar dilution (AD), broth microdilution (BMD) and polymyxin NP. Open Forum Infect Dis 2017;4:596

26 Simar S, Sibley D, Ashcraft D, Pankey G. Evaluation of the rapid polymyxin NP test for polymyxin B resistance detection using Enterobacter cloacae and Enterobacter aerogenes isolates. J Clin Microbiol 2017;55:3016-3020

27 Pragasam AK, Shankar C, Veeraraghavan B, et al. Molecular mechanisms of colistin resistance in Klebsiella pneumoniae causing bacteremia from india-a first report. Front Microbiol 2017;7:2135

28 Kumar M, Saha S, Subudhi E. More furious than ever: escherichia coli-acquired co-resistance toward colistin and carbapenems. Clin Infect Dis 2016;63(9):1267-1268

29 Kumar A, Biswas L, Omgy N, et al. Colistin resistance due to insertional inactivation of the mgrB in Klebsiella pneumoniae of clinical origin: first report from India. Rev Esp Quimioter 2018;31(5):406-410Us recate simint, cum quos 